MATEC Web of Conferences 13, 02030 (2014)

DOI: $10.1051 /$ matecconf/ 20141302030

(C) Owned by the authors, published by EDP Sciences, 2014

\title{
The Effect of Sodium Hydroxide on Drag Reduction using a Biopolymer.
}

\author{
Harvin Kaur A/P Gurchran Singh ${ }^{1, a}$, Dr. Azuraien Jaafar ${ }^{2}$ and Dr. Suzana Yusup ${ }^{3}$ \\ 1,2,3 Mechanical Engineering Department, Universiti Teknologi PETRONAS, Tronoh, Ipoh, Perak.
}

\begin{abstract}
Drag reduction is observed as reduced frictional pressure losses under turbulent flow conditions and hence, substantially increases the flowrate of the fluid. Practical application includes water flooding system, pipeline transport and drainage system. Drag reduction agent, such as polymers, can be introduced to increase the flowrate of water flowing, reducing the water accumulation in the system and subsequently lesser possibility of heavy flooding. Currently used polymer as drag reduction agents is carboxymethylcellulose, to name one. This is a synthetic polymer which will seep into the ground and further harm our environment in excessive use of accumulation. A more environmentally-friendly drag reduction agent, such as the polymer derived from natural sources or biopolymer, is then required for such purpose. As opposed to the synthetic polymers, the potential of biopolymers as drag reduction agents, especially those derived from a local plant source, are not extensively explored. The drag reduction of a polymer produced from a local plant source within the turbulent regime will be explored and assessed in this study using a rheometer where a reduced a torque produced can be perceived as a reduction of drag. The cellulose powder was converted to carboxymethylcellulose (CMC) by etherification process using sodium monochloroacetate and sodium hydroxide. The carboxymethylation reaction then was optimized against concentration of $\mathrm{NaOH}$. The research is structured to focus on producing the biopolymer and also assess the drag reduction ability of the biopolymer produced against concentration of sodium hydroxide.
\end{abstract}

\section{Introduction}

\subsection{Drag Reduction}

Drag reduction is a phenomenon where turbulent friction of a fluid can be greatly reduced (over $70 \%$ ) with the addition of small amount of additives (e.g. a few parts per million) (Darbouret et. al., 2009). The main purpose of using drag reduction is to reduce energy consumption by using active agent known as DRA without changing the mechanical parts of the process such as size of pumps, pipes and fittings (Bari et. al., 2010).

The experiment was conducted by Henaut et al. (2009) to demonstrate that the effectiveness of drag reduction agents can be assessed by comparing the flow curves of a treated solution (with DRA) to untreated solution (no DRA present). Jaafar et al. (2009) then proved that drag reduction is observed in what is termed as "Non-Newtonian flow". It can be clearly seen in 1 that the region where

${ }^{\text {a }}$ Harvin Kaur A/P Gurchran Singh: harvingurchran@gmail.com

This is an Open Access article distributed under the terms of the Creative Commons Attribution License 2.0, which permits unrestricted use, distribution, and reproduction in any medium, provided the original work is properly cited. 
drag reduction is detected is bounded by the drag reduction envelope, which are the Prandtl-Karman law for Newtonian turbulent flow and the maximum drag reduction asymptote by Virk (1975).

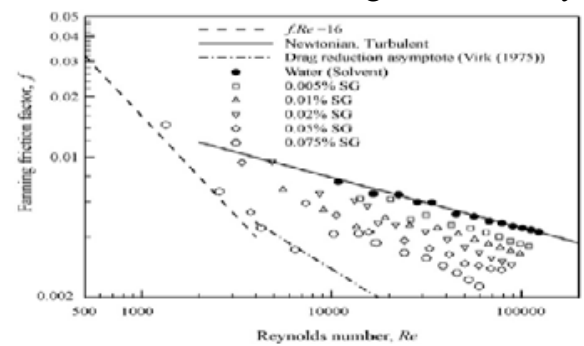

Figure 1: f-Re data for various Scleroglucan concentrations (Jaafar et. al., 2009) ${ }^{[4]}$

\subsection{Drag Reduction Agent (DRA)}

Toms (1949) was the first to report that drag reduction phenomenon and observed that the addition of few parts per million of long-chain polymers in a turbulent flow produces a dramatic reduction of the friction drag. This phenomenon has been the subject of extensive reviews by Mowla and Naderi (2006), Ling and Hassan (2006), Li et al. (2007), Wan et al. (2008), Safri and Bouhadef (2008), Riccoa and Quadrio (2008), Bari and Yunus (2009) and many others.

Polymers are divided into two categories: synthetic polymers and natural polymers. Synthetic polymers are derived from petroleum oil, while natural polymers can be extracted from resources in nature. Although synthetic polymers possess good mechanical properties and thermal stability when used as flow improvers in pipelines, these materials biodegrade very slowly, which is an environmental concern. Moreover, for similar molecular weights, synthetic polymers are more expensive than natural polymers. On the other hand, natural polymers are biodegradable and easily obtained because they are produced in the form of carboxymethylcellulose or polysaccharides by microorganisms and plants.

Banana is an example of a fruit that contains a high amount of natural polymers. Banana peels, representing $40 \%$ of the total weight of fresh bananas (Tchobanoglous et al. 1993), have been underutilized. One way to utilize this waste is to synthesize the banana peel and convert it into Carboxymethylcellulose. Therefore, the author will use the Cavendish green banana peels for the project. The findings from the experiment will prove whether the Cavendish green banana peels is a good Drag Reduction Agent. Synthesizing the biopolymer will be shown in the next section.

\section{Methodology}

The Methodology for this research has been divided into two parts which are synthesizing the Biopolymer and conducting the rheometer study using the biopolymers produced as a Drag Reducing Agent (DRA).

\subsection{Synthesizing the Biopolymers}

Banana Peel is rinsed with water until clean and then sun-dried for 4 days. It will be grinded and then cooked with $\mathrm{NaOH}$ at $100^{\circ} \mathrm{C}$. Black slurry will then be obtained. It will be filtered and washed with water. The residue will then be dried in an oven at $55^{\circ} \mathrm{C}$ to constant weight for 24 hours, and the pulp would be produced. It will be washed with water, filtered and then the pulp will be dried again at $55^{\circ} \mathrm{C}$ for 24 hours. The dried pulp will be grounded again into powder with size below $1 \mathrm{~mm}$ and stored in polyethylene bags. Preparation of CMC sample consists of two reactions which are alkalization and carboxymethylation reaction. Alkalization reaction begins after adding $\mathrm{NaOH}$ into $15 \mathrm{~g}$ of pure cellulose and isopropanol under mechanical stirring for an hour. Then, carboxymethylation reaction start once the sodium monochloroacetate (SMCA) is added while the reaction continuously stirs at 400rpm. Reaction time and $\mathrm{NaOH}$ concentration are controlled in this reaction. The mixture is then filtered and suspended in $100 \mathrm{~mL}$ of methanol overnight. The slurry was neutralized using glacial 
acetic acid. The samples undergo washing process using $70 \%$ ethanol solution for four times to remove undesired product. Lastly, the sample was dried in the oven at $55^{\circ} \mathrm{C}$ temperature. The parameter that was varied is the concentrations of sodium hydroxide $(\mathrm{NaOH})$.

\subsection{Rheometer study}

Unlike other experiment set ups as discussed in most literatures on Drag Reduction, the AR-G2 DCC rheometer from TA Instruments is chosen as the experiment instrument for this research as precise data can be obtained and recorded. A double concentric cylinder is used to set in the rheometer for this study. The inner cylinder will be rotating while the outer one will be attached to the base of the rheometer. The Reynolds number corresponding to this geometry, Re, is given by

$$
R e=\frac{\Omega \rho R_{m}(\Delta R)}{\mu}
$$

where: $\Omega$ : Angular velocity $(\mathrm{rad} / \mathrm{s}), \rho$ : Fluid density $\left(\mathrm{kg} / \mathrm{m}^{3}\right), R_{m}$ : Radius of rotating cylinder of the rheometer $(\mathrm{m}), \mathrm{R}_{3}, \Delta R$ : Radius difference between the outer and inner cylinder $(\mathrm{m}), \mathrm{R}_{4}-\mathrm{R}_{3}$, Fluid viscosity (Pa.s)

Due to the centrifugal force, the flow fields developed in the two parts of the geometry is different. For the case of the outer cylinder rotating, the centrifugal force tends to stabilize the flow field. The flow field of a Newtonian liquid becomes unstable when the dimensionless Reynolds is higher than about $50000^{[2]}$. For the case of the inner cylinder rotating, the centrifugal force contributes to a destabilization of the flow field. For a Newtonian liquid, the point at which the streamlines cease to be circular and at which the flow field presents Taylor instabilities has been found by Chhabra \& Richardson (1999) ${ }^{[1]}$ to take place for a critical Reynolds number defined by:

where:

$$
R e_{c}=\frac{\Omega \rho R_{m}(\Delta R)}{\mu}>58.3\left(\frac{R_{m}}{\Delta R}\right)^{0.5}
$$

\subsubsection{Sample Preparation}

For sample preparation (eg. water + DRA of $100 \mathrm{ppm}$ concentration),

$$
\frac{x}{x+y} \times 100 \%=\frac{100}{1 \times 10^{6}} \times 100 \%
$$

where: $\mathrm{x}=$ mass of commercial DRA (gram), $\mathrm{y}=$ mass of water (gram)

In order to make sure that the DRA mixed well in water, an overhead stirrer was used with constant low speed (approximately $200 \mathrm{rpm}$ ) for the duration of 3 hours until the polymer solutions appeared to be visibly homogeneous. Once mixing was completed, the solution was sealed to avoid water loss by evaporationSolutions were left for at least 8 hours before rheological tests were conducted. Sample was loaded into the DCC The temperatures for all the DRA assessments were set to $25^{\circ} \mathrm{C}$. The gap was set to 2000 micron. The solution was then left to rest for 2 minutes in the DCC before rheological tests were conducted. Drag Reduction test was performed from $0 \mathrm{rad} / \mathrm{s}$ to $300 \mathrm{rad} / \mathrm{s}$ with $20 \mathrm{sample}$ points taken. The torque over the angular velocity will then be calculated to measure the drag reduction efficiency. The drag reduction percentage, $\%$ DR can be calculated using Equation (6).

$$
\% \mathrm{DR}=\frac{\mathrm{T}_{\mathrm{u}}-\mathrm{T}_{\mathrm{t}}}{\mathrm{T}_{\mathrm{u}}} \times 100
$$

where the subscripts $u$ and $t$ refer to the untreated fluid (without DRA) and treated fluid (with DRA).

\section{Results and Discussion}

\subsection{Solubility}

Experiments on the biopolymer were done varying the concentration of sodium hydroxide during the synthesizing of the biopolymer. The reaction time was 150 minutes and the reaction temperature was $55^{\circ} \mathrm{C}$ which remains constant. The concentrations of $\mathrm{NaOH}$ used for the experiment were $20 \mathrm{w} / \mathrm{v} \%$, $40 \mathrm{w} / \mathrm{v} \%$ and $60 \mathrm{w} / \mathrm{v} \%$ respectively. All three samples were mixed and left overnight for de-aeration. Then, it was tested using the rheometer on Day 1, Day 3, Day 7 and Day 14. The experiments were conducted using 100ppm of biopolymer. 


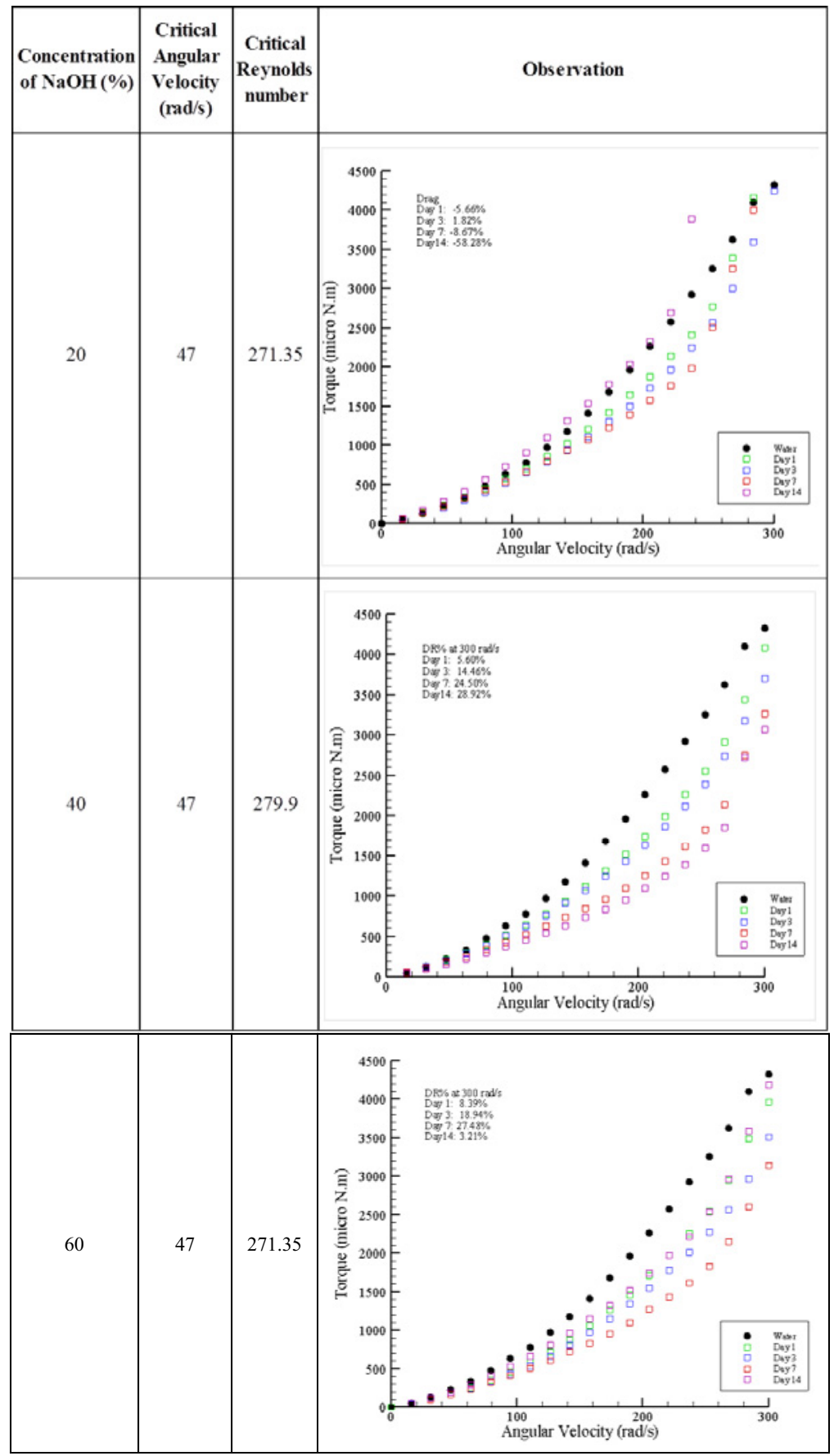

Figure 2: Effect of Concentration of Sodium Hydroxide on Drag Reduction

The $\mathrm{NaOH}$ concentration plays a great role in determining the production of CMC and the by-product. The proportion of $\mathrm{NaOH}$ must be at optimum to obtain high value of degree of substitution. It is observed on day 1 when the test was conducted, the drag reduction percentage increases steadily with the increment of concentration of $\mathrm{NaOH}$. However, in sample 2, throughout the 14 days when the test was conducted, it was observed that there was no decrement in drag reduction percentage. This shows 
that the $\mathrm{NaOH}$ increased the solubility of the mixture. However, in sample 3, when the concentration of $60 \%$ was tested, it was observed that after day 7 , the drag reduction percentage started to decrease. This shows that the optimum condition for the carboxymethylation of cellulose is at $40 \mathrm{w} / \mathrm{v} \%$. The higher the increment of $\mathrm{NaOH}$ concentration, the more the by-product of sodium glycolate will form as compared to CMC. Hence, the environment created will determine either main or side reaction is more dominant.

Furthermore, there was no drag produced on day 1 for sample 1 as $20 \mathrm{w} / \mathrm{v} \%$ concentration of $\mathrm{NaOH}$ is too low. Sodium hydroxide dissolves in water easily and it helps the mixture get dissolved. In contrast to that, higher $\mathrm{NaOH}$ concentration will decrease the viscosity of the CMC solution. This is due to the side reaction which takes place and results in the formation of sodium glycolate from the monochloroacetic acid. The carboxymethylation process which converts the sodium chloroacetate to sodium glycolate is a side reaction that will occur. The sodium glycolate formed will increase if the concentration of $\mathrm{NaOH}$ becomes higher. This is due to the monochloroacetic acid molecules tend to react with the $\mathrm{NaOH}$ which leads to the destruction of cellulose chains or CMC polymer. From the results, it can be observed that the increasing of $\mathrm{NaOH}$ concentrations affected the increase of percent yield of CMC. However, the yield of $\mathrm{CMC}$ is highest at $40 \%$ of $\mathrm{NaOH}$ concentrations. According to Varshney (2010), this observation could corroborate substitution of carboxymethyl group from carboxymethylation because of the higher molecular weight than hydroxyl group of carboxyl group.

The yield of $\mathrm{CMC}$ increases with increasing concentration of $\mathrm{NaOH}$ and attained the best drag reduction of $8.39 \%$ on day 1 at an alkali concentration of $60 \%(\mathrm{w} / \mathrm{v})$. This finding could be explained by considering the carboxymethylation procedure, where two competitive reactions took place simultaneously. The first involved reaction of the cellulose hydroxyl with Monohloroacetic acid in the presence of $\mathrm{NaOH}$ to give $\mathrm{CMC}$. The second reaction involves the conversion of monochloroacetate to sodium glycolate as byproduct by reacting with $\mathrm{NaOH}$ (Kirk and Othmer, 1967) ${ }^{[9]}$.

\section{Conclusion}

In conclusion, from the results shown, Carboxymethylcellulose could be synthesized from Banana peel by carboxymethylation. Using a DCC rheometer to evaluate DRA shows great potential in replacing the current method which is the flow loop. The study of the performance on the biopolymer made from banana peel has shown great characteristics as a drag reduction agent and results which are added into water that will serve to reduction of pumping power losses in the oil and gas industry and reduction in energy consumption. Experimental results showed that the presence of biopolymers in water helps in the performance of DRA. Solubility also increases when the percentage of $\mathrm{NaOH}$ increases.

\section{References}

1. Chhabra, R.P. and Richardson, J.F., "Non-newtonian flow in the process industries" Fundamentals and Engineering. (1999)

2. Darbouret, M., Henaut, I., Palermo, T., Hurtevent, Ch., Glenat, P., $14^{\text {th }}$ International Conference on Multiphase Technology. (2009)

3. Henaut, I., Darbouret, M., Palermo, T., Glenat, P., Hurtevent, C., Proceedings of SPE International Symposium on Oilfield Chemistry. (2009)

4. Jaafar, A., Escudier, M.P., Poole, R.J., Journal of Non-Newtonian Fluid Mechanics, 161, 86-93. (2009)

5. Mowla, D., Naderi, A., Chemical Engineering Science, 61, 1549 - 1554. (2005)

6. Munson et. al., "Fundamentals of Fluid Mechanics".John Wiley \& Sons (Asia) Pte Ltd., 45-55. (2010)

7. Toms, B.A., "Some observation on the flow of linear polymer solutions through straight tubes at large Reynolds number", Proceedings of the First International Congress on Rheology, Pt. 2, North-Holland, Amsterdam, 135. (1948)

8. Virk, P.S., "Drag reduction fundamentals", AIChE J. 21 (4) Vol. 21, 189. (1975)

9.Ganji, S., K.M. Chinnala and J. AukunuruPreparation, excipient properties and pharmacological activities of okra mucilage. Pharmacog. Maga., 4: 73-77. (2008)

10. N.M. Ismail, A.Bono, Optimization of Reaction Conditions for preparing Carboxymethylcellulose. (2010)

11. R.S. Pornchai Rachtanapun, Synthesis of Carboxymethyl Cellulose from Durian Husk. (2005) 